\title{
Achados oculares em pacientes com mielomeningocele:
} 72 casos

\author{
Ocular findings in 72 patients with meningomyelocele
}

\author{
Mônica Fialho Cronemberger ${ }^{(1)}$ \\ Marcia Keiko Uyeno Tabuse ${ }^{(2)}$ \\ Luis Tibiriça Aguilar ${ }^{(3)}$ \\ Marcelo Fernandes da Costa ${ }^{(4)}$ \\ Ivan Ferraretto ${ }^{(5)}$
}

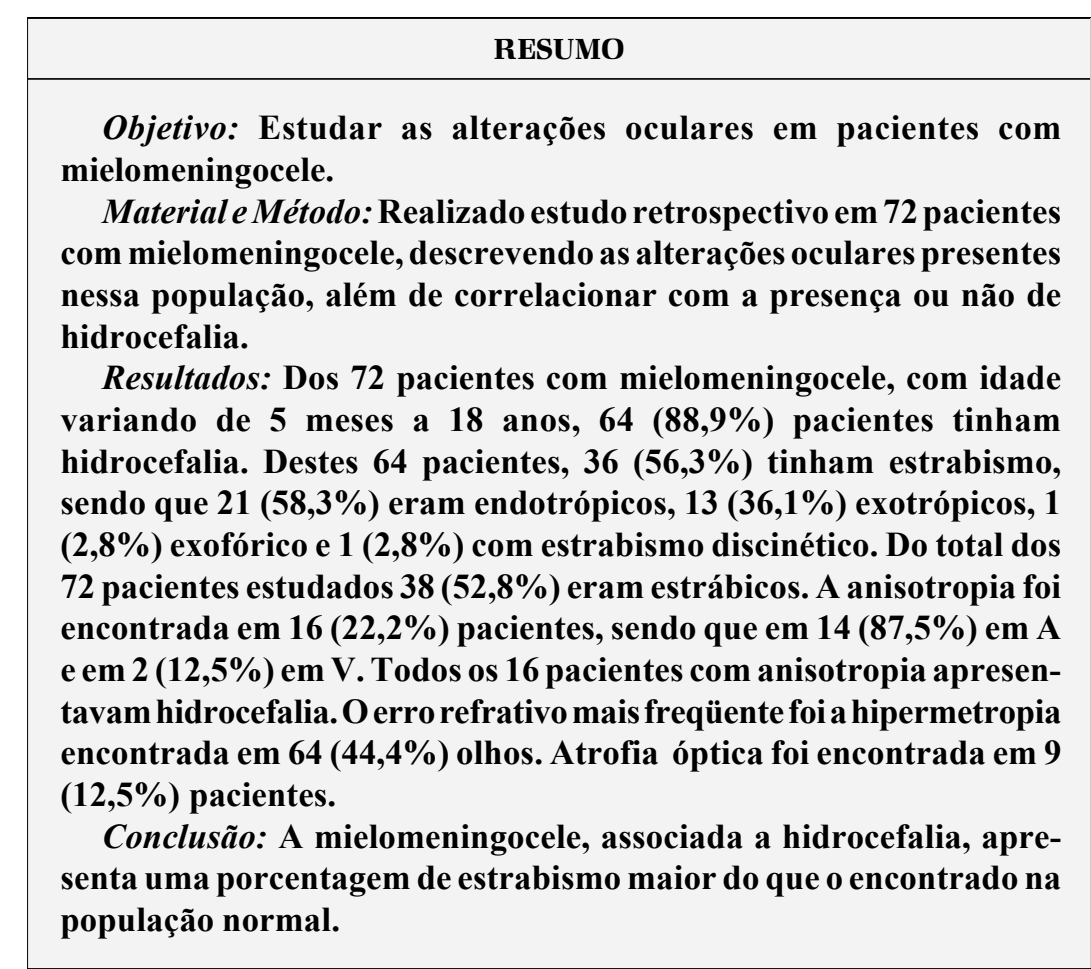

Palavras-chaves: Mielomeningocele; Hidrocefalia; Estrabismo.
Trabalho desenvolvido na Associação de Assistência à Criança Defeituosa (A.A.C.D.).

(1) Médica oftalmologista da Associação de Assistência à Criança Defeituosa (A.A.C.D.), médica voluntária da UNIFESP.

(2) Chefe do setor de oftalmologia da A.A.C.D., Doutora pela UNIFESP.

Médico oftalmologista da A.A.C.D., médico voluntário da UNIFESP.

(4) Ortoptista da A.A.C.D.

(5) Diretor Clínico da A.A.C.D.

Endereço para correspondência: Mônica Fialho Cronemberger - Rua: Lacedemonia 587 Apt 73A Aeroporto - São Paulo (SP) CEP: 04634-020.

\section{INTRODUÇ̃̃̃O}

Mielomeningocele é um defeito de desenvolvimento que se manifesta como uma falha na fusão completa dos arcos vertebrais da coluna vertebral ${ }^{1,2}$. Isto resulta em um crescimento displásico da medula e das meninges. O defeito é causado por uma falha primária no fechamento do tubo neural. A mielomeningocele lombar baixa resulta de um defeito no fechamento na porção posterior do tubo neural baixo, durante a $4^{\mathrm{a}}$ semana de gestação ${ }^{1}$.

Mielomeningocele é o tipo mais severo de espinha bífida, mas avanços recentes como: descoberta de novos antibióticos, progressos na medicina como controle da hidrocefalia (neurocirurgia), permitiram maior sobrevida à maioria destas crianças, que atualmente, ultrapassam a primeira infância, conseguindo chegar alguns casos à idade adulta ${ }^{3,4}$. Esta maior sobrevida condiciona o aparecimento de seqüelas importantes que os levam a procurar os Centros de Reabilitação e clínicas especializadas ${ }^{3}$.

Pacientes com mielomeningocele nos quais ocorre a hidrocefalia, a incidência de estrabismo é maior que na população normal ${ }^{5}$. 
Neste trabalho vamos descrever as alterações oculares presentes nessa população, além de correlacionar com a presença ou não de hidrocefalia. Não encontramos na literatura nacional nenhum outro trabalho sobre este tema.

\section{PACIENTES, MATERIAIS E MÉTODOS}

Estudo retrospectivo de 72 prontuários de pacientes com diagnóstico de mielomeningocele, atendidos no setor de Oftalmologia-Ortóptica da Associação de Assistência à Criança Defeituosa - A.A.C.D. , no período de janeiro de 1997 a março de 1999. As crianças tinham idades que variavam de 5 meses a 18 anos; $43(59,7 \%)$ eram do sexo feminino e 29 $(40,3 \%)$ eram do sexo masculino.

A mielomeningocele foi classificada segundo o nível de acometimento e pesquisado presença ou não de hidrocefalia.

\section{Exame Oftalmológico}

O exame oftalmológico incluiu: exame externo, medida da acuidade visual, biomicroscopia, motilidade ocular, retinoscopia sob cicloplegia e oftalmoscopia indireta. A biomicroscopia foi realizada em todos os pacientes com mais de 12 meses que já possuiam um controle suficiente do pescoço.

A medida da acuidade visual foi realizada pelos cartões de Acuidade de Teller nos pacientes não verbais e não cooperativos $(N=24)$ ou pelos testes com optotipos (lineares "E" de Snellen ou isolados Light House) realizados nos restantes $(\mathrm{N}=26)$. Os restantes $(\mathrm{N}=22)$ tiveram apenas uma avaliação qualitativa (comportamento visual com luz e objetos coloridos). Estes pacientes passaram em consulta oftalmológica e não retornaram até o momento para avaliação quantitativa (Teller). O resultado de todas as medidas de acuidade visual foram transformados em logMAR e comparados com normas para idade descritas previamente por Salomão \& Ventura $(1995){ }^{6}$ e Mayer et al (1995) ${ }^{7}$. Ambliopia foi determinada pela comparação das diferenças de acuidade interocular com as normas descritas para os cartões de Acuidade de Teller ${ }^{6,7}$. Para os testes com optotipos uma diferença interocular $>0,10$ $\log$ MAR foi determinada para diagnóstico de ambliopia.

No exame da motilidade ocular foram utilizados os métodos padronizados ${ }^{8}$, para medida dos desvios e rotações binoculares. A hiperfunção variou de $+1 \mathrm{a}+4$ e a hipofunção de $-1 \mathrm{a}-4$.

No exame de refração todas as crianças foram cicloplegiadas com 1 gota de colírio anestésico de cloridrato de proximetacaína $0,5 \%$ (Alcon), 1 gota de colírio de tropicamida 1\% (Alcon), 1 gota de colírio de ciclopentolato 1\% (Allergan-Frumtost). A refração era feita após 30 a 40 minutos de instilação.

$\mathrm{Na}$ oftalmoscopia indireta a palidez do nervo óptico foi pesquisada.

Método estatístico utilizado: teste z (teste para proporção) ${ }^{9}$.

\section{RESULTADOS}

A mielomeningocele nível lombar foi a mais freqüente $(48,6 \%)$, sendo neste grupo o nível lombar alto $(54,3 \%)$ o mais evidente. Em segundo lugar está o grupo de mielomeningocele nível torácico $(34,7 \%)$.

A hidrocefalia estava presente em 64 pacientes $(88,9 \%)$.

Nos pacientes em que foi possível uma medida quantitativa da acuidade visual $(\mathrm{N}=50)$, a ambliopia foi detectada em $5(10,0 \%)$ pacientes. Neste grupo onde foi realizada a medida quantitativa da acuidade visual (A.V.) obtivemos A.V. $\geq$ $20 / 50$ (Snellen) ou 0,40 (logMAR) em $53(53,0 \%)$ olhos e $<$ $20 / 50$ (Snellen) ou 0,40 (logMAR) em $47(47,0 \%)$ olhos.

No exame externo, as alterações mais encontradas foram: base nasal larga em $32(44,4 \%)$ pacientes, epicanto em 16 $(22,2 \%)$ pacientes, assimetria facial em $2(2,8 \%)$ e telecanto em $1(1,4 \%)$ paciente.

No exame da biomicroscopia tivemos apenas 1 paciente com blefarite no olho esquerdo e outro com hordéolo interno na pálpebra superior do olho esquerdo.

Dos 72 pacientes examinados 38 (52,8\%) eram estrábicos. Sendo que destes: 22 (57,9\%) pacientes tinham endotropia, 13 $(34,2 \%)$ tinham exotropia, $2(5,3 \%)$ exoforia e $1(2,6 \%)$ tinha estrabismo discinético.

Nas endotropias, que foi o maior grupo encontrado, o tamanho do desvio mais freqüente foi o leve ( + ou $-20 \Delta)$ e o moderado $(20 \Delta-30 \Delta)$, cada um destes grupos com 9 pacientes, totalizando 18 pacientes dos 22 endotrópicos.

A anisotropia mais encontrada foi a incomitância alfabética em A em 14 (19,4\%) pacientes. Todos com exceção de 1, tinham hiperfunção de músculo oblíquo superior em ambos os olhos variando de $+1 \mathrm{a}+3$. Destes 14 pacientes na posição primária do olhar: $10(71,4 \%)$ pacientes tinham endotropia, 3 $(21,4 \%)$ tinham exotropia e $1(7,1 \%)$ era ortotrópico. A anisotropia em V foi observada em $2(2,8 \%)$ pacientes. Ambos com hiperfunção de músculo oblíquo inferior de +1 . Na posição primária do olhar: $1(50,0 \%)$ tinha endotropia e o outro $(50,0 \%)$ exotropia. Todos os pacientes que apresentavam anisotropia tinham hidrocefalia.

Dos 8 pacientes que não possuiam hidrocefalia, 2 (25\%) tinham estrabismo, sendo que 1 paciente apresentava microendotropia $(<8 \Delta)$ e 1 paciente uma exoforia. A relação entre pacientes com mielomeningocele segundo a presença de estrabismo e a presença de hidrocefalia estão na Tabela I.

\begin{tabular}{|lccc|}
\hline Tabela I. & $\begin{array}{c}\text { Pacientes com mielomeningocele segundo a presença de } \\
\text { estrabismo e a presença de hidrocefalia. } \\
\text { Estrabismo }\end{array}$ \\
\cline { 2 - 4 } Hidrocefalia & Com & Sem & Total \\
Com & 36 & 28 & 64 \\
Sem & 2 & 6 & 8 \\
Total & 38 & 34 & 72 \\
Teste $z ;$ Pest $=5 \% ; 36 / 64=56.25 \%$ & & \\
estatística $z=18.812(p<0.0001)$ & & \\
\hline
\end{tabular}


O nistagmo estava presente em $12(16,7 \%)$ pacientes: sendo que em $1(8,3 \%)$ era manifesto tipo sacádico, em 1 $(8,3 \%)$ paciente era uniocular (olho esquerdo) tipo pendular e em $10(83,3 \%)$ pacientes o nistagmo era presente apenas nas posições extremas.

$\mathrm{O}$ erro refrativo mais freqüente foi a hipermetropia em 64 $(44,4 \%)$ olhos, em segundo lugar o astigmatismo hipermetrópico composto em $30(20,8 \%)$ olhos e em teceiro lugar o astigmatismo misto em 17 (11,8\%) olhos.

No exame de fundo de olho 9 (12,5\%) pacientes apresentavam palidez de papila. Todos estes 9 pacientes apresentavam hidrocefalia.

\section{DISCUSSÃO}

A incidência de mielomeningocele varia de uma região para outra. No Brasil não existe estatística mas nos E.U.A. é de aproximadamente 1 para cada 1000 nascidos vivos ${ }^{1,3}$. Pode-se detectar, através da amniocentese precoce, a presença de alfa-fetoproteína no líquido amniótico liberada da meningomielocele que não é recoberta por pele. O diagnóstico pode ser auxiliado pela ultra-sonografia e/ou radiografia ${ }^{10}$. Quanto ao sexo existe uma ligeira predominância pelo sexo feminino ${ }^{11}$, na proporção de $1: 1,5^{10}$.

Em $60 \%$ dos casos o defeito se localiza na região lombar ${ }^{3}$. No nosso estudo o nível lombar foi o mais freqüente, porém com uma porcentagem um pouco menor $48,6 \%$.

Hidrocefalia ocorre em $90 \%$ das crianças com mielomeningocele ${ }^{1}$. Esta freqüência está de acordo com o nosso levantamento que foi de $88,9 \%$. A hidrocefalia pode não estar presente ao nascimento, mas sinais clínicos desta condição podem se desenvolver até 2 ou 3 semanas. Freqüentemente muitos casos de hidrocefalia são diagnosticados e tratados com menos de 1 mês de vida ${ }^{1}$.

Biglan ${ }^{2}$ (1995) estudando 298 pacientes com espinha bífida encontrou $183(61,0 \%)$ estrábicos, $63,0 \%$ com esotropia e $37,0 \%$ com exotropia. Esta porcentagem está bem próxima da encontrada em nosso grupo onde: $38(52,8 \%)$ pacientes eram estrábicos, sendo a endotropia presente em $57,9 \%$ dos pacientes e a exotropia em $34,2 \%$. Sabendo-se que na população normal o número de estrábicos varia de $2 \%$ a $5 \%{ }^{12}$, o grande número de estrábicos encontrados na população com mielomeningocele pode ser explicado pelo fato de haver associadamente malformações de desenvolvimento do sistema nervoso central, incluindo hipoplasia ou aplasia de núcleos de nervos cranianos ${ }^{13}$. A porcentagem de estrábicos entre os pacientes portadores de mielomeningocele e hidrocefalia $(56,25 \%)$ é estatisticamente maior do que a proporção de estrábicos entre as pessoas normais.

Alguns autores especulam que uma hiperfunção de músculo oblíquo superior, especialmente em casos de pacientes com mielomeningocele associado com Arnold-Chiari tipo II, possa ter um substrato neuroanatômico localizado na região cerebelar e cervico-medular ${ }^{14}$.
Na literatura a anisotropia em A, no grupo de mielomeningocele, é mais freqüente nas exotropias ${ }^{1}$. Este dado vai contra o encontrado neste trabalho, onde a anisotropia em A foi mais freqüente nas endotropias.

Biglan ${ }^{1}$, em 1990, descreveu que $16 \%$ dos seus pacientes tinham algum grau de atrofia do nervo óptico. No nosso trabalho encontramos uma porcentagem de apenas $12,5 \%$. A atrofia do nervo óptico está associada com aumento da pressão intacraniana e papiledema; os quais são causas evitáveis de perda de visão, caso operados precocemente.

\section{CONCLUSÃO}

A mielomeningocele, associada a hidrocefalia, apresenta uma porcentagem de estrabismo maior do que o encontrado na população normal.

\section{SUMMARY}

Purpose: To study ocular findings in patients with meningomyelocele.

Methods: A retrospective study of 72 patients with myelomeningocele was performed to evaluate ocular motility disorders, refractive errors and indirect ophthalmoscopy findings.

Results: In a group of 72 patients with myelomeningocele, aged 5 month -18 years, 64 (88.9\%) had hydrocephalus. Of this group of 64 patients, 36 (56.3\%) had strabismus, 21 (58.3\%) of whom were esotropic, and 13 (36.1\%) exotropic, $1(2.8 \%)$ exophoric and $1(2.8 \%)$ presented sometimes esotropia and sometimes exotropia. Of the total of 72 studied patients, $38(52.8 \%)$ had strabismus. Anisotropia was found in $16(22.2 \%)$ patients, 14 (87.5\%) with an A pattern and 2 (12.5\%) with a V pattern. The most frequent refractive error was hyperopia, found in 64 (44.4\%) eyes. All the 16 patients with anisotropia had hydrocephalus. Atrophy of the optic nerve was found in 9 (12.5\%) patients.

Conclusion: Myelomeningocele associated with hydrocephalus had a higher percentage of strabismus than the normal population.

Keywords: Myelomeningocele; Hydrocephalus; Strabismus.

\section{REFERÊNCIAS BIBLIOGRÁFICAS}

1. Biglan AW. Ophthalmologic complications of meningomyelocele: a longitudinal study. Trans Am Ophthalmol Soc 1990;88:389-462.

2. Biglan AW. Strabismus associated with meningomyelocele. J Pediatr Ophthalmol Strabismus 1995;32(5):309-14.

3. Lianza S. Medicina de Reabilitação. Guanabara Koogan. p.296-313.

4. Lennerstrand G, Gallo JE. Neuro-Ophthalmological evaluation of patients with myelomeningocele and Chiari malformations. Dev Med Child Neurol 1990; 32(5):415-22.

5. Houtman WA, Meihuizen-De Regt Mj, Rutgers C. Strabismus and Meningomyelocele. Doc Ophthalmol 1981;50(2):255-61. 
6. Salomão SR, Ventura DF. Large sample population age norms for visual acuities obtained with Vistech - Teller acuity cards. Invest Ophthalmol Vis Sci 1995;36(3):657-70.

7. Mayer DL, Beiser AS, Warner AF, Pratt EM, Raye KN, Lang JM. Monocular acuity norms for the Teller acuity cards between ages one month and four years. Invest Ophthalmol Vis Sci 1995;36(3):671-85.

8. Prieto-Díaz J, Souza-Dias C. Estrabismo Roca 2" edición p.122-45.

9. Bussab WO, Morettin PA. Estatística Básica Atual 1987 p. 252.

10. Jones KL. Padrões reconhecíveis de malformações congênitas. Manole. $5^{\text {a }}$ edição p.608-9;721-2.
11. Werner D. Guia de deficiências e reabilitação simplificada. Coordenadoria Nacional para integração da pessoa portadora de deficiência (CORDE) 1994 p.167-174

12. Lyle TK, Bridgeman GIO. The binocular reflexes and the treatment of strabismus. Worth and Chavesse's squint, 1959.

13. Lennerstrand G, Gallo JE, Samuelsson L. Neuro-ophthalmological findings in relation to CNS lesions in patients with myelomeningocele. Dev Med Child Neurol 1990;32(5):423-31.

14. Hamed LM, Maria BL, Quisling RG, Mickle JP. Alternating skew on lateral gaze. Neuroanatomic pathway and relationship to superior oblique overaction. Ophthalmology 1993;100(2):281-6.

\title{
THIRDINIERNATIONALSYMPOSIUMON OPHIHAMOLOGY INTHEDEVELOPINGWORID
}

\author{
March 23-25, 2001 \\ Holiday Inn Financial District \\ San Francisco, California - USA
}

\section{Course Description:}

This two-and one-half-day symposium will bring together internationally recognized experts to discuss current issues in global blindness, including the diagnosis, prevention, and treatment of the major causes of blindness world wide. The focus of the course will be on the Developing World but causes of blindness in the developed world will be address as well. The course is intended for ophthalmologists, general physicians, nurses, public health workers, health administrators, policy makers, and health planners.

Contact Information: Karen Baranick

Medical Conference Planners, Inc.

1251 PostRoad

Scarsdale, NY 10583 USA

Tel.: (914)722-0664

Fax: (914)722-0465

email:medconfs@aol.com

web site:hHtp://www.medconfs.com 